\title{
Guest Editorial: Large-Scale Multimedia Content Analysis on Social Media
}

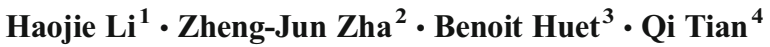

Published online: 2 February 2016

(C) Springer Science+Business Media New York 2016

Recent years have witnessed the emerging and booming of social platforms (e.g., Facebook, Instagram, YouTube, and Flickr etc.), where people record, share/broadcast, and comment on media content, i.e., images and videos, leading to an accelerated proliferation of social media on the Internet. The rapid increase of social media has raised numerous new research challenges to multimedia content analysis. On the other hand, the availability of massive social media has also presented many exciting opportunities and challenges to attack in particular within the field of multimedia content analysis.

This special issue aims to report recent research on large-scale social multimedia analysis and presents papers dealing with the various topics in this field. After rigorous reviewing, 11 papers were accepted for publication in MTAP. These papers cover most aspects for social media analysis. In particular, the papers of this issue concern (i) feature description and indexing for social media content, (ii) semantic understanding of social media, (iii) efficiency strategy for large scale practical social media analysis systems, (iv) social multimedia enabled applications, (v) social image processing and finally (vi) evaluations for social video content analysis. They are briefly discussed as follows.

Haojie Li

hjli@dlut.edu.cn

Zheng-Jun Zha

junzzustc@gmail.com

Benoit Huet

Benoit.Huet@eurecom.fr

Qi Tian

qitian@cs.utsa.edu

1 Dalian University of Technology, Dalian, China

2 University of Science and Technology of China, Hefei, China

3 Institut Eurecom, Biot, France

4 University of Texas at San Antonio, San Antonio, TX, USA 
Image feature description is the fundamental for social image analysis and the efficacy of feature description directly affects the performance of image retrieval, annotation and recognition. In the article "Fuzzy Bag of Visual Words for Social Image Description" (10.1007/ s11042-014-2138-4), Li et al. propose to utilize the fuzzy set theory to model and measure the ambiguity between the image features and visual words in codebook in the Bag of Visual Words representation. A new fuzzy membership function is designed and a genetic algorithm is proposed to obtain its optimal parameters. In article "Binary Code Re-ranking for Largescale Image Retrieval" (10.1007/s11042-014-2087-y), the authors address the issue of feature indexing in large scale social image retrieval. Two image ranking methods, i.e., distance weights based re-ranking and bit importance based re-ranking methods are developed to rerank the hashing indexed images for the given query. These methods assign high weights to important bits and small weights to less important bits, thus achieve better retrieval performance than traditional hashing approaches based on Hamming distance.

Social multimedia is characterized with the vast volume of data and the diversity of content even for the same category, which bring great challenges to the content understanding research and, scalable pattern analysis and machine learning techniques for social media are demanding. In article "Visual Concept Detection of Web Images based on Group Sparse Ensemble Learning" (10.1007/s11042-014-2179-8), the authors propose to collect large scale training data based on dictionary coherence to cover a wide variety of samples to handle the huge intraclass variations for visual concept detection. To efficiently train large scale data, they also propose a group sparse ensemble learning approach based on Automatic Group Sparse Coding. The article "Active Learning SVM with Regularization Path for Image Classification" (10.1007/s11042-014-2141-9) reports the recent work of active learning which is useful in image retrieval and classification. Observing the model parameters of active learning support vector machine are closely related to the training set, Sun et.al. argue that they should be dynamically adjusted instead of being kept fixed and, propose a novel approach to fit the entire solution path of SVM for every value of model parameters. While the article "Salient object detection and classification for stereoscopic images" (10.1007/s11042-014-2142-8) describes visual content analysis for a particular kind of media, stereoscopic images, which have become more and more prevalent following the rapid advances in 3D capturing and display techniques. Specifically, an iterative method that can mutually boost salient object detection and object classification is proposed for stereoscopic images.

Efficiency is critical in large scale social media content analysis and two accepted articles present good practice on this problem. The first article, "Realtime and Robust Object Matching with a Large Number of Templates" (10.1007/s11042-014-2305-7), presents a novel template based object matching approach based on improved Dominant Orientation Templates (DOT). The authors first propose a compact representation for DOT to greatly reduce the size of feature vector and then propose a fast partial occlusion handling strategy to boost the robustness of DOT. The second article "Large-Scale Paralleled Sparse Principal Component Analysis" (10.1007/s11042014-2004-4) reports an efficient and paralleled method of sparse Principal Component Analysis using graphics processing units (GPUs). The parallel GPU implementation is up to 11 times faster than the corresponding CPU implementation, showing significant advantage for large data analysis.

User generated content, available in massive amounts on the Internet, enable many potential applications. The article "Event-based Cross Media Question Answering" (10.1007/s11042-014-2085-0) targets at the representation of events using multimedia 
data. The authors present a framework for leveraging social media data to extract and illustrate social events automatically on any given query. Nowadays, social media has shown its predictive power in various domains. In the article "Predicting Movie BoxOffice Revenues by Exploiting Large-Scale Social Media Content" (10.1007/s11042-0142270-1), the authors use the crowd wisdom of social media, especially the posts of users, to predict movie box-office revenues. Both linear regression model and Support Vector Regression are employed for the prediction.

Some work focuses on the processing of social media. The article "Spatially guided local Laplacian filter for nature image detail enhancement" (10.1007/s11042-014-2058-3) addresses the enhancement of nature images in social media. Specifically, an improved local Laplacian filter that spatially guides the filtering strength by approximating the richness of image details is proposed. The proposed method endows the Laplacian filter with the ability to dynamically assign appropriate parameter values to different image content.

Along with the emerging focus of community-contributed videos on the web, there is a strong demand of a well-designed web video benchmark for the research of social network based video content analysis. The article "On Application-Unbiased Benchmarking of Web Videos from a Social Network Perspective" (10.1007/s11042-014-2245-2) releases such a benchmark named MCG-WEBV 2.0, which crawls 248,887 YouTube videos and their corresponding social network structure with 123,063 video contributors. The benchmark can be used to explore the fusion between content and network for several web video analysis tasks.

We thank all authors for their contributions to this special issue. We would also like to thank to all the reviewers for their hard work in judging and improving the quality of the submitted papers.

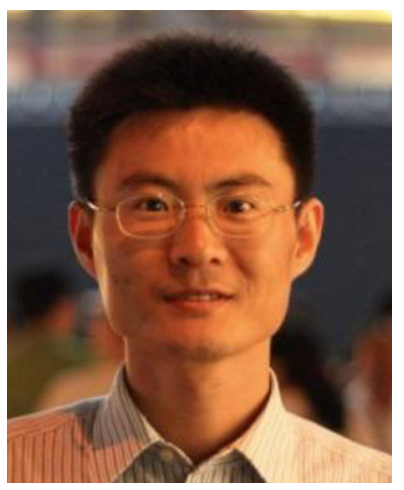

Haojie Li is a Professor in the School of Software, Dalian University of Technology. His research interests include social media computing and multimedia information retrieval. He has co-authored over 50 journal and conference papers in these areas, including IEEE TCSVT, TMM, ACM Multimedia, ACM ICMR, etc. He severed as a Guest Editor for Multimedia Tools and Applications. He has been serving as a technical program committee member or session chair for dozens of international conferences and a reviewer for over 10 prestigious international journals. He received the Best Paper Award in the ACM International Conference on Internet Computing and Services (ACM ICIMCS) 2011 and the 9th Joint Conference on Harmonious Human Machine Environment (HHME 2013).

Dr. Li received the B.E. and the $\mathrm{Ph}$. D. degrees from Nankai University, Tianjin and the Institute of Computing Technology, Chinese Academy of Sciences, Beijing, in 1996 and 2007 respectively. From 2007 to 2009, he was a Research Fellow in the School of Computing, National University of Singapore. He is a member of IEEE and ACM. 


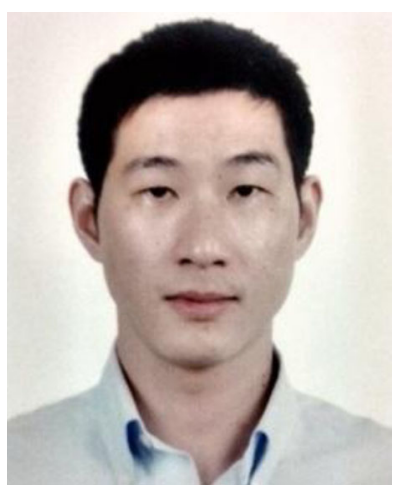

Zheng-Jun Zha is a Professor with University of Science and Technology of China, Hefei, China. His current research interests include multimedia big data computing and computer vision. He has published over 100 referred papers in these areas. He was the recipient of several paper awards from prestigious multimedia conferences, including the Best Paper Award in ACM Multimedia 2009, the Best Demo Runner-Up award in ACM Multimedia 2012, the Best Student Paper Award in ACM Multimedia 2013, the Best Paper Award in ICIMCS 2013, and the Best Paper Candidate Award in PCM 2014 etc. He served as a guest editor, organizer, and area chair for many prestigious international journals and conferences.

Dr. Zha received the B.E. degree (2004) in automation and the Ph.D. degree (2009) in pattern recognition and intelligent system from the University of Science and Technology of China (USTC), Hefei, China, respectively. He worked as a Senior Research Fellow (2011-2013) and Research Fellow (2009-2010) in the School of Computing, National University of Singapore (NUS).

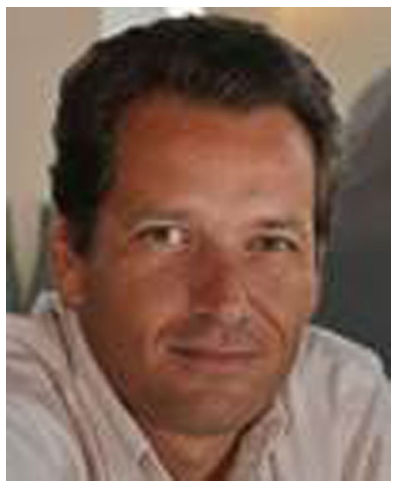

Benoit Huet is Assistant Professor in the multimedia information processing group of Eurecom (France). He received his BSc degree in computer science and engineering from the École Supérieure de Technologie Électrique (Groupe ESIEE, France) in 1992. In 1993, he was awarded the MSc degree in Artificial Intelligence from the University of Westminster (UK) with distinction, where he then spent two years working as a research and teaching assistant. He received his DPhil degree in Computer Science from the University of York (UK) for his research on the topic of object recognition from large databases. He was awarded the HDR (Habilitation to Direct Research) from the University of Nice Sophia Antipolis, France, in October 2012 on the topic of Multimedia Content Understanding: Bringing Context to Content. He is associate editor for IEEE Multimedia, Multimedia Tools and Application (Springer), Multimedia Systems (Springer) and ITE Transactions on Media Technology and Applications and has been guest editor for a number of special issues (IEEE Multimedia, IEEE trans. on Multimedia, Springer Multimedia Tools and Application, EURASIP Journal on Image and Video Processing, Elsevier Ecological Informatics, etc..). He regularly serves on the technical program committee of the top conferences of the field (ACM MM/ICMR, IEEE ICME/ICIP). He has co-authored over 150 papers in Books, Journals and International conferences. He is regularly invited to serves as reviewer for prestigious scientific journals as well as expert for project proposal at national, European and International level. He was 
the conference chair of the International Conference on Multimedia Modeling (MMM'2009) and the co-chair of the Pacific-rim Multimedia Conference (PCM'2014). He sits in the Steering Committee of the Multimedia Modeling conference. His current research interests include Large Scale Multimedia Content Analysis, Mining and Indexing - Multimodal Fusion - Socially-Aware Multimedia.

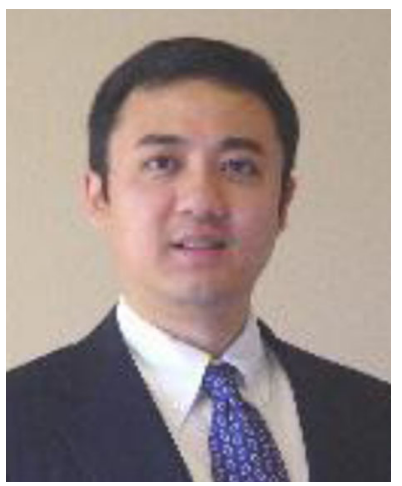

Qi Tian is currently a Full Professor in the Department of Computer Science, the University of Texas at San Antonio (UTSA). He was a tenured Associate Professor from 2008 to 2012 and a tenure-track Assistant Professor from 2002 to 2008. During 2008-2009, he took 1-year Faculty Leave at Microsoft Research Asia (MSRA) as Lead Researcher in the Media Computing Group to work on large-scale image search. He was a Visiting Scholar at MIAS center of UIUC in 2007 and a Visiting Professor in NEC Laboratories of America in 2003.

Dr. Tian received his Ph.D. in ECE from University of Illinois at Urbana-Champaign (UIUC) in 2002 and received his B.E. in Electronic Engineering from Tsinghua University in 1992 and M.S. in ECE from Drexel University in 1996, respectively. Dr. Tian's research interests include multimedia information retrieval, computer vision, pattern recognition and bioinformatics and published over 180 refereed journal and conference papers. He was the co-author of a Best Paper in MMM 2013, a Best Paper in ACM ICIMCS 2012, a Top 10 \% Paper Award in MMSP 2011, a Best Student Paper in ICASSP 2006, and co-author of a Best Paper Candidate in PCM 2007.

Dr. Tian has served as founding member of International Steering Committee for ACM International Conference on Multimedia Retrieval (ICMR, 2009-2014), ACM Multimedia Conference Review Committee Member (2009-), and International Steering Committee Member for ACM MIR (2006-2010), Best Paper Committee Member for ACM Multimedia 2009, ICME 2006 and 2009. He has served as general chair, program coordinator, and program chair for various international conferences including ACM Multimedia 2015, ACM Multimedia 2009, ACM CIVR 2010, ACM ICMCS 2009, MMM 2010, IMAI 2007, VIP 2007, 2008, MIR 2005 , etc. He has also served in various organization committees as Panel and Tutorial Chair, Publicity Chair, Special Session Chair, Track Chair in numerous ACM and IEEE conferences such as ACM Multimedia, VCIP, PCM, CIVR, ICME, and served as TPC members for prestigious conferences such as ACM Multimedia, SIGIR, $\mathrm{ICCV}$, and $\mathrm{KDD}$. 